\title{
DIRECT EVIDENCE FROM SPITZER FOR A LOW-LUMINOSITY AGN AT THE CENTER OF THE ELLIPTICAL GALAXY NGC 315
}

\author{
Q.-S. Gu, ${ }^{1,2}$ J.-S. HuAng, ${ }^{2}$ G. Wilson, ${ }^{3}$ and G. G. FazIO ${ }^{2}$ \\ Received 2007 September 15; accepted 2007 October 31; published 2007 November 16
}

\begin{abstract}
We present Spitzer Space Telescope InfraRed Array Camera (IRAC) and Multiband Imaging Photometer (MIPS) observations of the elliptical galaxy NGC 315. After removal of the host galaxy's stellar emission, we detected for the first time an infrared-red nucleus in NGC 315. We measured the spectral energy distribution (SED) for this active nucleus with a wavelength range from radio to X-ray, and obtained a bolometric luminosity of $L_{\mathrm{bol}} \approx 1.9 \times 10^{43} \mathrm{ergs} \mathrm{s}^{-1}$, corresponding to an extremely low Eddington ratio $L / L_{\mathrm{Edd}}$ of $4.97 \times 10^{-4}$. Our results confirm that the physical nature of the nucleus of NGC 315 is a low-luminosity AGN, consistent with recent optical and Chandra X-ray observations.
\end{abstract}

Subject headings: galaxies: active — galaxies: elliptical and lenticular, $\mathrm{cD}-$

galaxies: individual (NGC 315) — galaxies: nuclei — infrared: galaxies

Online material: color figures

\section{INTRODUCTION}

The recent discovery of a tight correlation between black hole $(\mathrm{BH})$ mass and the bulge's mass of the host galaxy suggests that the creation and growth of central black holes are an integral part of the formation of galactic bulges (Kormendy \& Richstone 1995; Kauffmann \& Heckman 2005). On the other hand, it also implies that supermassive black holes (SMBHs) are ubiquitous at the centers of elliptical galaxies. Although indeed most host galaxies of radio-loud and the brightest quasars at high redshift are elliptical galaxies (Hutchings \& Morris 1995; Bahcall et al. 1996; Falomo et al. 2005), the majority of SMBHs in nearby ellipticals are not very active (Ho et al. 1997), and therefore hardly detectable.

The low state of SMBHs in nearby ellipticals is most probably due to the lack of enough fuel supply-cold gas inflow into the central engine. It is generally accepted that elliptical galaxies are spheroidal distributions of older stars and lack substantial cold intersteller material (ISM). However, an $\mathrm{H} \alpha+$ $[\mathrm{N}$ II] narrowband imaging survey has shown that elliptical galaxies certainly have plenty of extended warm $\left(\sim 10^{4} \mathrm{~K}\right)$ gas (Shields 1991). By detecting positive signal from co-added images of the Infrared Astronomy Satellite (IRAS) survey scans, Knapp et al. (1989) discovered that $\sim 50 \%$ of optically selected ellipticals with visual magnitude brighter than $V=$ 14 contained cool interstellar matter. Temi et al. (2004) estimated that 16 elliptical galaxies observed with the Infrared Space Observatory (ISO) have dust masses on average 10 times greater than those previously detected by IRAS. Most recently, Kaneda et al. (2005) detected even polycyclic aromatic hydrocarbon (PAH) emission features in four elliptical galaxies with the Spitzer Infrared Spectrograph (IRS). Recent neutral hydrogen observations also show that plenty of extended gas is clearly present in early-type galaxies (Morganti et al. 2006; Noordermeer 2006 and references therein). All these results suggest that the presence of a significant amount of cold/warm

\footnotetext{
${ }^{1}$ Department of Astronomy, Nanjing University, Nanjing 210093, China; qsgu@nju.edu.cn.

${ }^{2}$ Harvard-Smithsonian Center for Astrophysics, 60 Garden Street, Cambridge, MA 02138.

${ }^{3}$ Spitzer Science Center, California Institute of Technology, MS 220-6, Pasadena, CA 91125.
}

interstellar gas at centers of elliptical galaxies is quite common, although the origin of such an amount of dust and cold gas is still uncertain (Caon et al. 2000).

The AGN signature of SMBHs has been seen in elliptical galaxies. In the recent Palomar spectroscopic survey, Ho et al. (1997) found that about $50 \%$ of elliptical galaxies show detectable emission-line nuclei, most of which $(\sim 87 \%)$ are classified as LINERs. Although it is still a subject of hot debate whether LINERs are genuine low-luminosity AGNs (see the recent review by Ho 2003), AGN activity is confirmed by radio and X-ray observations (Terashima et al. 2002; Filho et al. 2004).

Typical AGNs are also strong infrared sources. Ho et al. (1997) found that about 55\% of elliptical galaxies with emission lines were detected by IRAS as having infrared emission. Due to the lower spatial resolution $\left(\sim 3^{\prime}-4^{\prime}\right)$ of $I R A S$, it is impossible to determine whether the far-infrared emission from elliptical galaxies arises from AGN point sources at the center, or from an extended dust distribution seen as a dust lane in optical images (Lauer et al. 2005). One example is NGC 315, a wellstudied elliptical galaxy classified as a LINER by Ho et al. (1997). The HST image shows a clear dusty disk with $2.5^{\prime \prime}$ diameter in its center (Verdoes Kleijn et al. 1999), and the galaxy is also detected by IRAS at 12, 25, 60, and $100 \mu \mathrm{m}$ with flux densities of $0.081,0.150,0.270$, and $0.706 \mathrm{Jy}$, respectively (IRAS Faint Source Catalogue; Moshir et al. 1990), corresponding to $L_{\mathrm{FIR}}=2.3 \times 10^{9} L_{\odot}$ at the distance of 65.8 Mpc (Ho et al. 1997). Clearly, a high-resolution infrared image of NGC 315 is required to determine whether its infrared emission comes from the central $2^{\prime \prime}$ region.

The Spitzer Space Telescope (Werner et al. 2004) has 2" spatial resolution in the mid-infrared $(3.6-8.0 \mu \mathrm{m})$ and $5^{\prime \prime}$ at $24 \mu \mathrm{m}$. This makes it possible to study the infrared emission from central regions of elliptical galaxies. In this work we present a study of the nuclear activity in the elliptical galaxy NGC 315 based on observations by the Infrared Array Camera (IRAC; Fazio et al. 2004) and the Multiband Imaging Photometer for Spitzer (MIPS; Rieke et al. 2004) aboard the Spitzer Space Telescope. This Letter is organized as follows: the IRAC and MIPS data reduction, flux calibration, and photometry are in $\S 2$. We present multiwavelength physical properties of the nucleus of NGC 315 in $\S 3$. 


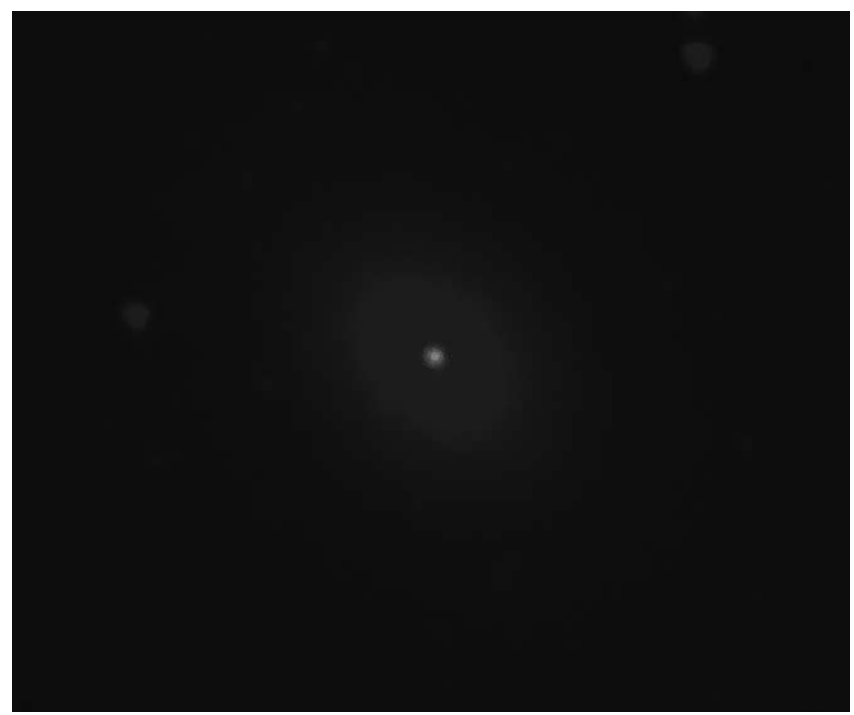

FIG. 1.-RGB false-color image of NGC 315, which combines IRAC 4.5 $\mu \mathrm{m}(\mathrm{B}), 5.8 \mu \mathrm{m}(\mathrm{G})$, and $8.0 \mu \mathrm{m}(\mathrm{R})$ images from the Spitzer Space Telescope. An infrared-red core is clearly seen in the central region. The image size is $2.0^{\prime} \times 2.0^{\prime}$. [See the electronic edition of the Journal for a color version of this figure.]

\section{DATA REDUCTION}

We downloaded the IRAC Basic Calibrated Data (BCD) for NGC 315 from the archive of the Spitzer Science Center (SSC). The BCD images had been performed with basic image processing, including dark subtraction, detector linearization corrections, flat-field corrections, and flux calibrations (see also the IRAC Data Handbook ${ }^{4}$ ). We further used the custom IDL software (Huang et al. 2004) to make the final mosaic images as shown in Figure 1. The MIPS image for NGC 315 was mosaicked by the SSC pipeline as post-BCD products. Both the IRAC and MIPS bands have absolute flux calibration accuracies of better than 10\% (Fazio et al. 2004; Rieke et al. 2004). Throughout this Letter, the magnitudes and colors were given in the $\mathrm{AB}$ system.

The surface photometry for NGC 315 was performed with the ellipse program in the ISOPHOT package of IRAF. ${ }^{5}$ The isophotal parameters, such as ellipticity and position angle, for NGC 315 were measured at $3.6 \mu \mathrm{m}$, where the signal-tonoise $(\mathrm{S} / \mathrm{N})$ ratio is highest. These parameters were then applied for the surface photometry at $4.5,5.8$, and $8.0 \mu \mathrm{m}$. IRAC photometry calibration is designed only for point sources. Recently Tom Jarrett provided additional aperture corrections for extended sources photometry in IRAC images. ${ }^{6}$ We applied this additional correction for the IRAC surface photometry of NGC 315. The total flux densities for NGC 315 in each IRAC

\footnotetext{
${ }^{4}$ Available at http://ssc.spitzer.caltech.edu/irac/dh/.

${ }^{5}$ IRAF is distributed by the National Optical Astronomy Observatory, which is operated by the Association of Universities for Research in Astronomy, Inc., under cooperative agreement with the National Science Foundation.

${ }^{6}$ See http://spider.ipac.caltech.edu/staff/jarrett/irac/calibration/index.html.
}

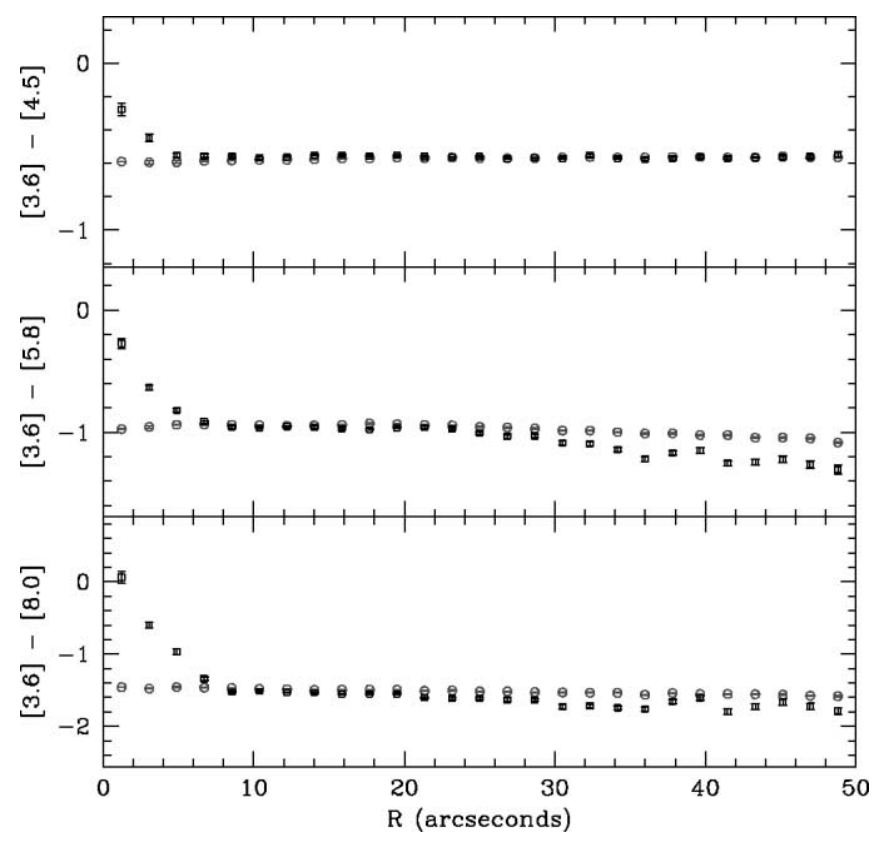

FIG. 2.-IRAC color distribution for NGC 315. [See the electronic edition of the Journal for a color version of this figure.]

band are given in Table 1. We diluted the 3.6, 4.5, and $5.8 \mu \mathrm{m}$ IRAC images so that they have the same spatial resolution as the $8.0 \mu \mathrm{m}$ image to obtain accurate IRAC color distributions for NGC 315.

Figure 2 shows three IRAC color distributions of [3.6] [4.5], [3.6] - [5.8], and [3.6] - [8.0] for NGC 315. At 5" < $R<50^{\prime \prime}$, these infrared colors are roughly constant and consistent with those of an M star (Pahre et al. 2004). The central region, however, shows a much redder color, indicating a nonstellar origin of the infrared emission. In Figure 2 we also plot the IRAC colors of a normal elliptical galaxy, NGC 5557, for comparison. NGC 5557 has not been identified as having any AGN signature in the center, and it does not show red IRAC colors in the central region $\left(R<5^{\prime \prime}\right)$. The constant color distributions for NGC 315 at $R>5^{\prime \prime}$ suggest a uniform stellar population, which is confirmed by the high-S/N long-slit optical spectrophotometry (see Fig. 3 in Cid Fernandes et al. 2005).

In the IRAC band the emission from the central region of NGC 315 consists of two components: stellar and nonstellar emission. However, we cannot interpolate the stellar distribution measured in the outer region to the region with $R<2^{\prime \prime}$, because the stellar distribution in the central region of an elliptical galaxy can have various profiles. Pahre et al. (2004) argued that the $3.6 \mu \mathrm{m}$ emission traces the stellar mass distribution very well (see also Wu et al. 2005); we relied on the IRAC $3.6 \mu \mathrm{m}$ image to remove the contribution from the underlying stellar population. We used the mean colors ([3.6] [4.5], [3.6] - [5.8], and [3.6] - [8.0]) at the region of $10^{\prime \prime}$ and $30^{\prime \prime}$ to scale the $3.6 \mu \mathrm{m}$ image to remove the contribution from the underlying stellar components in the $4.5,5.8$, and $8.0 \mu \mathrm{m}$

TABLE 1

IRAC FLUXES FOR NGC 315

\begin{tabular}{ccccc}
\hline \hline Component & $\begin{array}{c}3.6 \mu \mathrm{m} \\
(\mathrm{mJy})\end{array}$ & $\begin{array}{c}4.5 \mu \mathrm{m} \\
(\mathrm{mJy})\end{array}$ & $\begin{array}{c}5.8 \mu \mathrm{m} \\
(\mathrm{mJy})\end{array}$ & $\begin{array}{c}8.0 \mu \mathrm{m} \\
(\mathrm{mJy})\end{array}$ \\
\hline Total $\ldots . . . .$. & $202.8 \pm 2.78$ & $120.8 \pm 2.12$ & $73.9 \pm 1.83$ & $52.3 \pm 1.56$ \\
Nucleus $\ldots . .$. & $2.424 \pm 0.291$ & $3.650 \pm 0.357$ & $5.870 \pm 0.453$ & $11.450 \pm 0.633$ \\
\hline
\end{tabular}



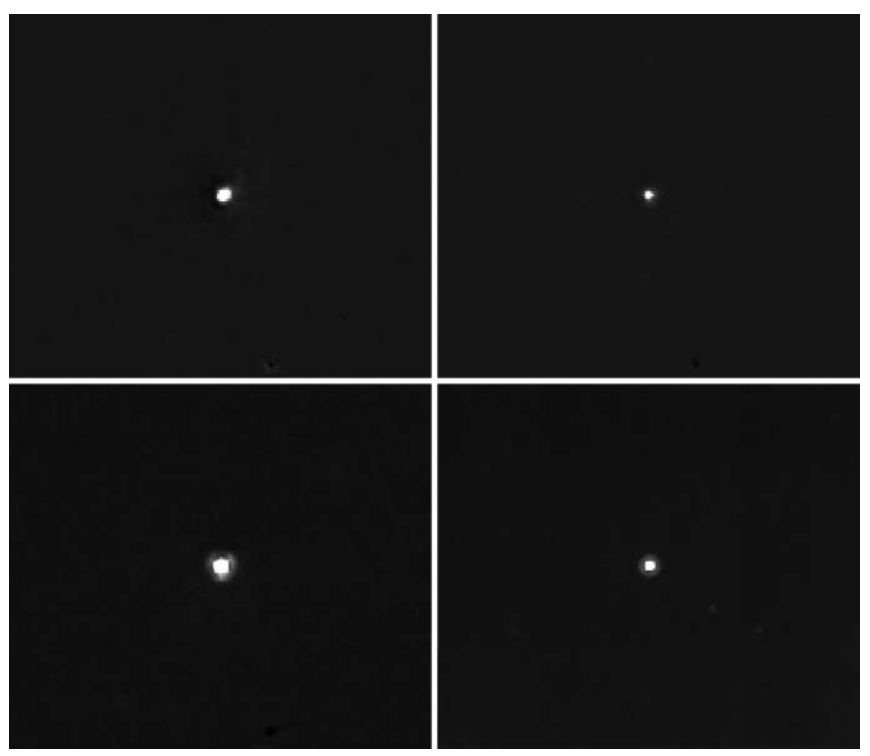

FIG. 3.-Residual images at 4.5 (top left), 5.8 (top right), and $8.0 \mu \mathrm{m}$ (bottom left) of NGC 315 after removing the underlying stellar contribution. The $24 \mu \mathrm{m}$ MIPS image is also shown in the bottom right panel. We can even see the ringlike structure in the residual $8.0 \mu \mathrm{m}$ image, which is exactly the characteristic feature of the IRAC $8.0 \mu \mathrm{m}$ point-spread function (PSF). [See the electronic edition of the Journal for a color version of this figure.]

images. There is only one point source left in all three residual images, as shown in Figure 3. The flux density for the point source is as follows:

$$
f_{\text {residual }}(\lambda)=f_{\text {ns }}(\lambda)-R_{\text {stellar }} \times f_{\text {ns }}(3.6),
$$

where $\lambda=4.5,5.8$, and $8.0 \mu \mathrm{m}, f_{\text {residual }}$ is the total flux for the point source in the residual images, $f_{\mathrm{ns}}$ is the flux density for the nonstellar red nucleus, and $R_{\text {stellar }}$ is the flux ratio of the stellar emission. We have to assume the shape of the SED for the central nonstellar emission to solve the four flux densities out of three equations. The flux density $f_{\text {residual }}(\lambda)$ appears as a power-law distribution, and it is reasonable to assume that $f_{\mathrm{ns}}(\lambda)$ is a power-law function. The $f_{\mathrm{ns}}$ for $3.6,4.5,5.8$, and $8.0 \mu \mathrm{m}$ are given in Table 1. The residual point source is also consistent with the MIPS $24 \mu \mathrm{m}$ image. NGC 315 appears as a point source at $24 \mu \mathrm{m}$ (Fig. 3) because its stellar emission is negligible in this band. Therefore for the first time we determine that all infrared emission from NGC 315 detected by IRAS and Spitzer comes from the central pointlike source.

\section{PHYSICAL PROPERTIES OF THE RED NUCLEUS}

At the resolution of IRAC it is not possible to tell whether the IR emission is really coming from the AGN nucleus, or from the dusty disk seen in the HST images. If the IR emission is produced by heated dust, we can estimate the dust temperature using the 8.0 and $24 \mu \mathrm{m}$ fluxes, since Spitzer Infrared Spectrograph (IRS) observations suggest that the PAH feature at $7.7 \mu \mathrm{m}$ is very faint or even absent in elliptical galaxies (Kaneda et al. 2005). Assuming a graybody radiation characterized by a dust emissivity going as $\lambda^{-1.5}$, we derive a dust temperature of $T_{\text {dust }} \sim 168 \mathrm{~K}$, which is similar to the dust temperatures measured in the central regions of Seyfert galaxies, $T_{\text {dust }} \sim 170 \mathrm{~K}$ (Deo 2007). We also need to check whether the amount of dust mass inferred from the optical dusty disk is consistent with the dust mass required to explain the IR emission. De Ruiter et al. (2002) estimated a dust mass from IRAS

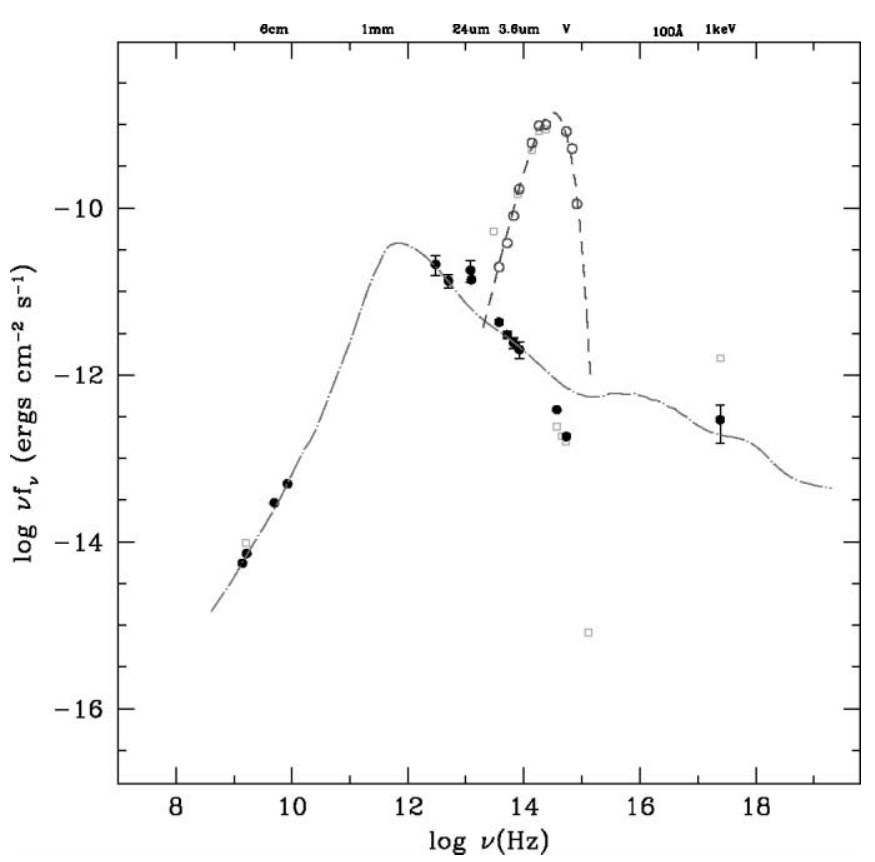

FIG. 4.- Spectral energy distribution of the infrared-red core in NGC 315. The SEDs of Sgr A* (dot-dashed line) and NGC 4261 (squares) are also shown and normalized to the $8.42 \mathrm{GHz}$ emission flux of NGC 315 . The SED of the host galaxy of NGC 315 is shown as circles, and is well fitted by blackbody radiation with a temperature of $3950 \mathrm{~K}$ (dashed line). [See the electronic edition of the Journal for a color version of this figure.]

data $\left(M_{\text {dust }} \sim 8.0 \times 10^{5} M_{\odot}\right)$ that is a thousand times larger than that inferred from the optical reddening absorption study $\left(M_{\text {dust }} \sim 8.0 \times 10^{2} M_{\odot}\right)$, which might indicate a significant contribution of IR emission from the central AGN. A ringlike structure is clearly visible in the $8.0 \mu \mathrm{m}$ image (Fig. 3, bottom left), which is very similar to the IRAC 8.0 point-spread function (PSF) first Airy ring, thus providing further evidence that the IR emission comes from the AGN nucleus. We conclude therefore that both the dusty disk and the central AGN contribute to the IR emission. With the current data set, we cannot address this further. For the future, IR variability data would certainly help to solve the current issue.

Venturi et al. (1993) presented multifrequency radio observations of NGC 315 with parsec resolution; they detected radio emission from a nucleus with a size of $0.05 \mathrm{pc}$ and $\nu L_{\nu}(5 \mathrm{GHz})$ $\approx 1.6 \times 10^{40} \mathrm{ergs} \mathrm{s}^{-1}$. Chandra also detected a pointlike nucleus, which is well fitted with a single power law with an intrinsic absorption of $n_{\mathrm{H}} \sim(7.6 \pm 1.2) \times 10^{21} \mathrm{~cm}^{-2}$, and an $0.5-8 \mathrm{keV}$ luminosity of $(7.6 \pm 0.3) \times 10^{41} \mathrm{ergs} \mathrm{s}^{-1}$ (Worrall et al. 2007). Thus, using the $H S T$ data and the accurate flux densities in the mid- and far-infrared derived in this Letter, we are able to extend the spectral energy distribution (SED) of the nucleus of NGC 315 from the radio to X-ray, as shown in Figure 4. Also shown in Figure 4 is the SED of the host galaxy, which is seen to be very well fitted by blackbody radiation of $3950 \mathrm{~K}$, which is consistent with its optical spectrum (Cid Fernandes et al. 2005) and the infrared colors derived in this Letter.

Integrating the SED we obtain a bolometric luminosity of $L_{\text {bol }}=1.93 \times 10^{43} \mathrm{ergs} \mathrm{s}^{-1}$ for the nucleus of NGC 315 . Denicolo et al. (2005) obtained the central stellar velocity dispersion of NGC 315 as $\sigma=246 \pm 8 \mathrm{~km} \mathrm{~s}^{-1}$, which according to the $M_{\mathrm{BH}^{-}} \sigma$ relation (Tremaine et al. 2002) corresponds to a central SMBH mass of $M_{\mathrm{BH}} \approx 3.1 \times 10^{8} M_{\odot}$. Combining the SMBH mass with the bolometric luminosity, we derive an Eddington 
ratio $L / L_{\mathrm{Edd}}$ of $4.97 \times 10^{-4}$, typical of low-luminosity AGNs (Ho 1999).

For comparison, we plotted in Figure 4 the SED of Sgr A*, the supermassive black hole at the Galactic center, which is well measured from radio to X-rays (Yuan et al. 2003). We find that the SED shape of NGC 315 is remarkably similar to that of Sgr $A^{*}$, which might suggest a similar accretion mode - a radiatively inefficient accretion disk-for both NGC 315 and Sgr A*. This is surprising because there are several systematic differences between Sgr A* and NGC 315: (1) $L / L_{\text {Edd }}$ of Sgr A* is only $3 \times 10^{-9}$ (Yuan et al. 2003), more than 5 orders of magnitude lower than that of NGC 315. (2) The X-ray spectrum of Sgr A* is much steeper than that of NGC 315, their photon indexes being 2.7 and 0.57 , respectively (Baganoff et al. 2003; Worrall et al. 2007). (3) We see in Figure 4 that the SED of NGC 315 deviates significantly from that of $\mathrm{Sgr} \mathrm{A}^{*}$ in the optical band. Since the HST data have very high spatial resolution, the good agreement in the IR band may be fortuitous since we cannot separate emission from the central dusty disk and AGN with the present IRAC data. The match may also be fortuitous in the radio band since NGC 315 has extended radio jets, which may contaminate the core emission (see Table 2 for core data).

In order to throw more light on this subject, we also plot in Figure 4 the SED of NGC 4261, which has a similar $L / L_{\text {Edd }}$ $\left(2.8 \times 10^{-5}\right)$ to NGC 315 . We see that NGC 4261 matches the SED of NGC 315 quite well in the radio and optical bands, but is more luminous in X-rays. We must conclude that the apparent match of SEDs does not provide a unique physical interpretation about the physics of radiation from AGNs.

In this work, we presented Spitzer IRAC and MIPS observations of the elliptical galaxy NGC 315. After careful removal of the dominant stellar light from the host galaxy, the IRAC revealed that all the nonstellar IR emission originates from a compact region in the center of NGC 315. Although there is a nonnegligible contribution from the central dusty disk to the IR emission of NGC 315, the ringlike pattern in the nonstellar $8.0 \mu \mathrm{m}$ image, the significant excess of dust mass inferred from IR data over that of the optical dusty disk, and the power-law distribution of the IRAC fluxes strongly suggest that we have direct evidence of a lowluminosity AGN in NGC 315, which might dominate the IR emission.
TABLE 2

DATA FOR THE CORE OF NGC 315

\begin{tabular}{|c|c|c|c|}
\hline & $\begin{array}{c}\nu \\
(\mathrm{Hz})\end{array}$ & $\begin{array}{c}\nu F_{\nu} \\
\left(\text { ergs } \mathrm{cm}^{-2} \mathrm{~s}^{-1}\right)\end{array}$ & Reference \\
\hline 1.40 & $\times 10^{9}$. & $5.54 \times 10^{-15}$ & 1 \\
\hline 1.66 & $\times 10^{9}$. & $7.23 \times 10^{-15}$ & 2 \\
\hline 4.99 & $\times 10^{9}$. & $2.93 \times 10^{-14}$ & 2 \\
\hline 8.42 & $\times 10^{9}$. & $4.95 \times 10^{-14}$ & 2 \\
\hline 1.25 & $\times 10^{13}$ & $1.39 \times 10^{-11}$ & 3 \\
\hline 2.97 & $\times 10^{13}$ & $2.82 \times 10^{-12}$ & 4 \\
\hline 3.75 & $\times 10^{13}$ & $2.84 \times 10^{-12}$ & 3 \\
\hline 5.17 & $\times 10^{13}$ & $1.49 \times 10^{-12}$ & 3 \\
\hline 6.67 & $\times 10^{13}$ & $8.95 \times 10^{-13}$ & 3 \\
\hline 8.33 & $\times 10^{13}$ & $5.71 \times 10^{-13}$ & 3 \\
\hline 5.40 & $\times 10^{14}$ & $1.83 \times 10^{-13}$ & 5 \\
\hline 3.68 & $\times 10^{14}$ & $3.86 \times 10^{-13}$ & 5 \\
\hline 2.42 & $\times 10^{17}$ & $2.902 \times 10^{-13}$ & 6 \\
\hline
\end{tabular}

REFERENCES. - (1) Capetti et al. 2005; (2) Venturi et al. 1993; (3) this work; (4) Heckman et al. 1983; (5) Verdoes Kleijn et al. 2002; (6) Worrall et al. 2007.

The authors are very grateful to the anonymous referee for her/ his constructive report which improved the Letter very much. We also thank Jorge Melnick, Matthew Ashby, and Luis Ho for thoughtful discussion, and Feng Yuan for providing us the SED of Sgr A*. This work has been supported by Program for New Century Excellent Talents in University (NCET) of China. Q. G. U. would like to acknowledge the financial support from the China Scholarship Council (CSC), the National Natural Science Foundation of China under grants 10221001 and 10633040, and the National Basic Research Program (973 program 2007CB815405). This research has made use of NASA's Astrophysics Data System Bibliographic Services and the NASA/IPAC Extragalactic Database (NED), which is operated by the Jet Propulsion Laboratory, California Institute of Technology, under contract with the National Aeronautics and Space Administration. This work is based on observations made with the Spitzer Space Telescope, which is operated by the Jet Propulsion Laboratory, California Institute of Technology, under NASA contract 1407.

\section{REFERENCES}

Baganoff, F. K., et al. 2003, ApJ, 591, 891

Bahcall, J. N., Kirkakos, S., \& Schneider, D. P. 1996, ApJ, 457, 557

Caon, N., Macchetto, D., \& Pastoriza, M. 2000, ApJS, 127, 39

Capetti, A., Verdoes Kleijn, G., \& Chiaberge, M. 2005, A\&A, 439, 935

Cid Fernandes, R., Gonzalez Delgado, R. M., Storchi-Bergmann, T., Martins, L. P., \& Schmitt, H. 2005, MNRAS, 356, 270

Denicolo, G., et al. 2005, MNRAS, 356, 1440

Deo, R. 2007, Ph.D. thesis, Georgia State Univ.

de Ruiter, H. R., et al. 2002, A\&A, 396, 857

Falomo, R., Kotilainen, J. K., Scarpa, R., \& Treves, A. 2005, A\&A, 434, 469

Fazio, G. G., et al. 2004, ApJS, 154, 10

Filho, M. E., Fraternali, F., Markoff, S., Nagar, N. M., Barthel, P. D., Ho, L. C., \& Yuan, F. 2004, A\&A, 418, 429

Heckman, T. M., et al. 1983, ApJ, 272, 400

Ho, L. C. 1999, ApJ, 516, 672

. 2003, in ASP Conf. Ser. 290, Active Galactic Nuclei: From Central

Engine to Host Galaxy, ed. S. Collin et al. (San Francisco: ASP), 379

Ho L. C., Filippenko, A. V., \& Sargent, W. L. W. 1997, ApJS, 112, 315

Huang, J.-S., et al. 2004, ApJS, 154, 44

Hutchings, J. B., \& Morris, S. C. 1995, AJ, 109, 1541

Kaneda, H., Onaka, T., \& Sakon, I. 2005, ApJ, 632, L83

Kauffmann, G., \& Heckman, T. M. 2005, Philos. Trans. R. Soc. London, A, 363,621
Knapp, G. R., Guhathakurta, P., Kim, D.-W., \& Jura, M. 1989, ApJS, 70, 329 Kormendy, J., \& Richstone, D. 1995, ARA\&A, 33, 581

Lauer, T. R., et al. 2005, AJ, 129, 2138

Morganti, R., et al. 2006, MNRAS, 371, 157

Moshir, M., et al. 1990, IRAS Faint Source Catalog (ver. 2.0; Greenbelt: NASA/ GSFC)

Noordermeer, E. 2006, Ph.D. thesis, Univ. Groningen

Pahre, M. A., et al. 2004, ApJS, 154, 235

Rieke, G. H., et al. 2004, ApJS, 154, 25

Shields, J. C. 1991, AJ, 102, 1314

Temi, P., Brighenti, F., Mathews, W. G., \& Bregman, J. D. 2004, ApJS, 151, 237

Terashima, Y., Iyomoto, N., Ho L. C., \& Ptak, A. F. 2002, ApJS, 139, 1

Tremaine, S., et al. 2002, ApJ, 574, 740

Venturi, T., et al. 1993, ApJ, 408, 81

Verdoes Kleijn, G. A., Baum, S. A., de Zeeuw, P. T., \& O’Dea, C. P. 1999 , AJ, 118, 2592 2002, AJ, 123, 1334

Werner, M. W., et al. 2004, ApJS, 154, 1

Worrall, D. M., et al. 2007, MNRAS, 380, 2

$\mathrm{Wu}, \mathrm{H}$. , et al. 2005, ApJ, 632, L79

Yuan, F., Quataert, E., \& Narayan, R. 2003, ApJ, 598, 301 